\title{
Research on the Construction of Precision Medical System Under the Background of Big Data-The Roles and Responsibilities of Government, Hospitals and Medical Workers
}

\author{
Xin Wang ${ }^{1,3 *}$, Lizhang $\mathrm{Xu}^{2,4}$, Bin $\mathrm{Liu}^{3}$, Fangxiang Zhang ${ }^{1}$ \\ ${ }^{1}$ Guizhou People's Hospital, Guiyang 550008, China \\ ${ }^{2}$ Key Laboratory of Advanced Manufacturing Technology, Guizhou University, Guiyang 550003, China \\ ${ }^{3}$ Huaxi Medical College, Sichuan University, Chengdu 610041, China \\ ${ }^{4}$ Industrial and Information Commission of Guiyang, Guiyang 550081, China
}

Corresponding Author Email: 110667637@qq.com

https://doi.org/10.18280/rces.080206

Received: 12 February 2021

Accepted: 23 May 2021

\section{Keywords:}

big data, precision medicine, system construction

\begin{abstract}
This article explores the roles and responsibilities of government, hospital and medical workers in the construction of precision medical system under the background of big data, which provide reference advices for setting out big data-related policies by the government, promoting the applications of big data technology in the medical field by the hospital, and using big data technology to help improve the efficiency of clinical diagnosis and treatment or make precise medical practice by medical workers. The main research contents are followed. It presents some problems and countermeasures in setting out big data-related policies by the government. This article studies the work tips of hospitals, as the main body of the implementation of the responsibility and obligation, and how to use big data technology in application. Meanwhile, it tries to analyze the problems and difficulties which hospitals and medical workers need to pay attention to applying big data technology in precision medicine.
\end{abstract}

\section{INTRODUCTION}

With the rapid development of large-scale biological sample database, genomics, medical technology, big data technology and computing power, precision medicine is becoming a focus of attention. Based on individual gene and health difference, it is possible to develop a new method to solve health disease, which will lead to a new medical era.

\section{OVERVIEWS}

\subsection{The concept of big data}

Big Data, or mega data, refers to the vast, high-growth, and diverse information assets that require new processing patterns to provide greater decision-making, insight, and process optimization. In the age of big data, by Victoor Myer Schönberg Im Stubaital and Kenneth Cooke, big data refers to the use of all data for analysis rather than the short cut of random sampling. 5V features of big data are Volume, Velocity, Variety, Value, Veracity. Yao et al. [1] have studied the thinking, difficulties and countermeasures of Hospital Management under the background of big data of health care, and advocated making full use of the massive data produced in the process of medical behavior and business processing, it is not only beneficial to the development of medical clinical research and public health, but also beneficial to the improvement of the management of medical profession. The collection, application and analysis of high-quality data is the premise and difficulty of scientific management of hospital by data, which needs to be solved by top-level design and overall arrangement, perfecting related policies, and strengthening professional training. Zhang and Chi [2] started from the problems of patients' privacy and medical information leakage risk and management risk, the difficulty of sharing big medical data, and the shortage of funds and talents in smart hospitals, focusing on ensuring patient privacy and medical information security, making full use of medical big data, building research and development platforms, joint medical associations and third-party resources, and building a multi-disciplinary workforce, it is proposed that the core of the future AI + smart hospital is to serve patients, strengthen the standardization of medical data sharing and management, and realize the goal of maximizing service efficiency. Li Houzhe and others have studied the application of Internet of things technology in intelligent hospital, Xue [4] has studied the integration and application of medical data under the background of hospital information, and so on.

\subsection{The concept of precision medicine}

There is no consensus on the definition of Precision Medicine, an emerging approach to disease prevention and management that takes into account individual genetic, environmental and lifestyle differences. In general, precision medicine is not simply "mobile medicine, " it is the result of a combination of precision bio-gene therapy and medical big data, based on a systematic approach, using big data analysis, a new medical model for patient-driven medical management. Precision medicine will undoubtedly promote the development of genomics, new management and big data analysis will also accelerate the biological and medical discovery. 
In 2008, Professor Clayton Christensen of the Harvard business school pioneered the concept of precision medicine. In 2011, the National Research Council formally introduced the concept of precision medicine in a paper titled Toward Precision Medicine: Building a knowledge network for new taxonomies of biomedicine and disease. In his 2015 state of the Union address, President Obama called for a "precision medicine program" that would spend $\$ 215$ million in fiscal year 2016 to promote personalized medicine.

\section{THE CONSTRUCTION OF PRECISION MEDICAL SYSTEM UNDER THE BACKGROUND OF BIG DATA}

In the construction of precision medical system under the background of big data, government, hospital and medical workers are the three key roles which are indispensable [3]. Overall, the current application of big data technology in the medical field is still gradually spread, the degree of application is far higher abroad than at home. And in the domestic hospital level using big data technology cases are very rare. It can be said that although "big data" has been widely used in many fields and industries, but in the health care industry has just started [3]. With the world into the era of big data is accelerating, who can make good use of big data platform, who can seize the opportunity for development. At present, taking Xiangya hospital as an example, which is at the forefront of big data application in China, the hospital takes "Xiangya famous medical project" as the carrier, takes the establishment of big data information platform as the core driving force, and establishes the big data electronic platform, strengthen the organic combination of clinical teaching and scientific research. Each of the Central South University's top doctors will be given a $\$ 1$ million research grant and full time assistants to create an electronic repository of information about the entire process of patient care, that is, doctors are interested in, think typical of a variety of data, including text, audio, video and so on all collected, set up a special electronic database, and from which to find the law. This has several advantages: first, the training of young doctors, electronic platform in the course of diagnosis and treatment of problems and solutions, as well as patients before and after a variety of comprehensive data, young doctors can use this platform selflearning, to grow quickly; Second, research can be done through the platform, and many things can be mined out through the sorting and integration of data. Third, crossdisciplinary study can be conducted to improve the level of clinical diagnosis and treatment, and various disciplines can find databases of related disciplines, from the relevant data analysis to analyze the pathology. The above experience and practice are worth learning.

According to the investigation, most of the top three hospitals in Guizhou Province have adopted electronic medical records, and the degree of information is increasing, but the application of big data technology has not started, so the application potential of big data technology is huge. However, we are also aware of the implementation of big data in the context of precision medicine is still not small difficulties.

\subsection{The role and responsibilities of government}

At present, the government has issued more policy documents related to big data. In August 2015, the State
Council issued the circular of the State Council on Printing and issuing the action plan for promoting the development of big data, in June 2016, the State Administration of Health State Council General Office issued the guiding opinions on promoting and standardizing the application and development of big data in health care. Taking Guiyang, Guizhou Province as an example, the province attaches great importance to the development of big data, it has been promoted to one of the three strategic actions of the province, namely "big poverty alleviation, big data and big ecology", sentence is too long, please supply a shorter sentence, clear Development Thinking and perfect implementation measures. However, it can still be seen from the national to local, for the precise medical segmentation policy is rare. In the context of big data in the construction of precision health care system, we suggest that the government should take the lead of the overall deployment and guidance of the responsibility to do a good top-level design, create a good atmosphere for development.

(1) To guide medical institutions and social enterprises to participate in the construction in a reasonable and orderly manner by means of policy support and financial support, and at the same time to do a good job in the introduction and training of talents;

(2) To establish medical big data standards and standardize data formats as soon as possible; Facilitate data receipt and sharing, reduce data cleaning costs, improve data utilization efficiency;

(3) Earnestly fulfill government responsibility, do a good job in data sharing, such as building a public data sharing platform to provide reasonable data sharing of government public resources, at the same time, we should strengthen data security and avoid data leakage, etc.

(4) We should increase publicity, raise society's awareness of precision medical treatment, and take an active part in the construction of precision medical treatment system.

\subsection{The role and responsibility of the hospital}

In China, hospitals at all levels and all kinds of hospitals have carried out the hospital information construction extensively since the middle and late 1980s. According to incomplete statistics, each domestic Grade III, Class A hospital invested about 0.2 to 400 million yuan, and an upward trend year after year. The basic establishment of medical services covered by the business information system, greatly improved the level of business information. The hospital information construction has played an important supporting role in improving the level of patient service, work efficiency, medical treatment quality and hospital operation. There are no less than 600 hospital-related information system service providers in China, and the number of hospital internal information systems varies from dozens to dozens, usually built and maintained by different information system service providers, but each service provider's information system from the development tool, the database structure, uses the code as well as the management mentality are all different, causes in the hospital each aspect information interconnection and the information sharing difficulty. At present, because of the decentralized storage and management of information, it cannot be fully shared [5]. When most hospital medical staff implement medical services, if a doctor's order is made, an application for an examination is made, the result of an examination is queried, the result of a physical examination is consulted, and the information of a health file is queried, etc., 
it must be completed in each sub-information system, and it is not convenient to obtain comprehensive and comprehensive information of a patient, some of the information is not even accessible. The integration and interoperability of the hospital information system is not high, and it has not achieved the desired effect in the aspects of patient-centered service, paramedic work, comprehensive analysis of the condition, rapid diagnosis, and shortening the time of patient visit [6]. Cuggia, M. and Combes, S analyse the French health data hub and the German medical informatics initiatives [7]. Venkatesh, A.N. studies Internet of medical things (IoMT), artificial intelligence (AI), machine learning (ML), big data, mobile apps and advanced sensors to remold the future of healthcare industry [8]. Other foreign researchers also from various aspects of this issue carried out relevant research [9-11]. However, the overall design of the information construction of most hospitals in China only focuses on meeting the selfdevelopment plans of hospitals in such aspects as medical services, teaching and research, and reducing medical expenses, it is difficult to realize information sharing and service coordination among hospitals and regions due to the failure to consider the overall development of population health information in regions and even countries. Lack of long-term development momentum and international competitiveness.

In the construction of precision medical system under the background of big data, hospital is the main battlefield of precision medical treatment, and has a long way to go. In the case of Guiyang, how to make full use of the resources of the local big data industry, especially how to build a Chinese "digital valley" to bring many data centers to the ground and the development of the crowdfunding industry to bring big data resources, etc. , to help the medical sector take the lead in such changes as informatization, data-based, networking and so on, and form a pilot demonstration effect nationwide; and to establish a sound policy system, it is worth thinking about how to effectively and orderly advance the development of the integration of big data and big health, especially in the medical field. We suggest that the hospital focus on the following aspects to do a good job:

(1) Emancipate the mind, fully recognize that precision medicine is the new trend of future medical development, make rational use of big data related technology, and promote the innovation and development of new diagnostic and treatment methods such as precision medicine and intelligent diagnosis;

(2) As the main battlefield to break the problem of isolated data island, hospitals should not only break the rule of law, but also have the courage to innovate, the foresight and the ability to carry out reform and innovation. Still Take Guiyang as an example, at present, most of the top three hospitals in Guiyang have a fair level of information, electronic medical records and other information-based means are more popular, digital means of diagnosis and treatment are more common, with a certain degree of precision medical implementation basis, but it is very common that the information system is not compatible with the scene. The whole process of a hospital's diagnosis and treatment involves multiple information systems, or part of the diagnosis and treatment has not been informationalized, it is common that all informatized data cannot be shared on the same platform. In the future development, it is suggested that the hospital level data opening and data sharing should be done step by step to encourage and support the compatible upgrading of different types of database management systems to avoid the negative impact of system barriers; To strengthen the protection of patient privacy in data opening and sharing, to break the narrow protection consciousness between departments and departments, to open the data channels of various parts, to avoid artificial interference, to facilitate data collection and data mining, and so on

(3) To strengthen the training of relevant talents, pay attention to the training of relevant knowledge and skills, and encourage and support medical personnel to improve relevant skills.

\subsection{Roles and responsibilities of health care workers}

Medical workers, especially clinical medical workers, are the vanguard and practitioners of precision medicine, the terminal link and an important link of the whole precision medical system, and its importance is self-evident. From the previous survey, medical workers have a high degree of acceptance of precision medicine, and it is not uncommon for hospitals, especially the third-class hospitals, to see a doctor repeatedly because of the high pressure of seeing a doctor, clinical medical workers are also eager to increase the means of diagnosis and treatment, especially the high accuracy of scientific diagnosis and treatment auxiliary means, so as to avoid the misdiagnosis rate, greatly improve the efficiency of diagnosis and treatment.

(1) in the construction of a precision medical system, medical workers should improve their ideological understanding, innovate their work concepts, pay attention to updating their knowledge system and fulfill the key tasks of the practitioners of precision medical treatment, so as to make full use of the convenience brought about by precision medical treatment, and to participate actively in the construction of precision medical system.

(2) in the precision medical system under the big data background, the data collection is an important link, but quite a lot of data collection is completed by the general medical worker, therefore, the medical worker enhances the sense of responsibility, completes the related data collection, it is the responsibility of medical workers to avoid ineffective collection, reduce redundant information, improve data quality, make efficient use of big data analysis results, use them reasonably and effectively, and help to realize personalized clinical diagnosis and treatment and personalized surgery efficiently.

\section{CONCLUSION}

Health care big data is an important fundamental strategic resource for the country. The application and development of big data in health care, represented by precision medicine, will bring about profound changes in the health care model, help stimulate the impetus and vitality of deepening the reform of the health care system, and improve the efficiency and quality of health care services, we will expand the supply of resources and continue to meet the diverse health needs of the people at various levels. Taking safeguarding the health of all the people as the starting point, we will strengthen top-level design, consolidate the foundation at the grassroots level, improve policies and systems, and create new working mechanisms. We will vigorously promote the interconnection, integration, openness and sharing of government health care information 
systems and public health care data, to eliminate the isolated island of information, actively create a development environment to promote the safety and innovative application of big data in health care, explore new service models and foster new business forms through "Internet + health care" , efforts to build a people-satisfied medical and health service will provide strong support for building a healthy China, completing the Xiaokang in all respects and realizing the Chinese dream of the Chinese Dream.

\section{ACKNOWLEDGMENT}

Support Fund: 1. "Research on the development of precision medicine based on big data technology", contract no.: QKH[2016]1532-3;

2. "Effect of modified nerve block combined with remazolid mesylate on the prognosis of elderly patients undergoing hip replacement", contract no.: BEIJING HONGYI MEDICAL DEVELOPMENT FOUNDATION.

\section{REFERENCES}

[1] Yao, D.M., Zhai, X.H., Mei, C.Z., Wang, D., Zhang, T. Guo, J.L., Xu, J.X., Zhu, J.S. (2021). Thinking, difficulties and countermeasures of Hospital Management under the background of big data of health care. China Hospital, 25(7): 28-30.

[2] Zhang, M.Y., Chi, W.W. (2021). Research on the status quo and development trend of artificial intelligence intelligent hospital. Chinese Journal of Library and Information of Traditional Chinese Medicine, 45(3): 4649.

[3] (2021). How to layout health care big data application in Shandong Province. Circular of the People's Government of Shanxi Province on Printing and Distributing the "Fourteenth Five-Year" New Business Form Planning of
Shanxi Province. Bulletin of Shanxi Provincial People's Government, 5: 8-26.

[4] Xue, Z. (2021) Application of Internet of things technology in smart hospital. Building Electrical Engineering, 40(5): 67-71.

[5] Jin, Z.H., Yang, L.X., Zhu, L., Wang, D.M. (2021). Integration and application of medical data under the background of hospital informatization. Microcomputer Application, 37(5): 116-118.

[6] Xu, D. (2021). Design of big data governance model for hospitals. Medical Information. Medical Information, 34(10): 18-20.

[7] Cuggia, M., Combes, S. (2019). The French health data hub and the German medical informatics initiatives: Two national projects to promote data sharing in healthcare. Yearbook of Medical Informatics, 28(1): 195-202. https://doi.org/10.1055/s-0039-1677917

[8] Venkatesh, A.N. (2019). Reimagining the future of healthcare industry through Internet of medical things (IoMT), artificial intelligence (AI), machine learning (ML), big data, mobile apps and advanced sensors. Artificial Intelligence (AI), Machine Learning (ML), Big Data, Mobile Apps and Advanced Sensors. https://doi.org/10.2139/ssrn.3522960

[9] (2019). Information Technology-Medical Informatics; Investigators at SungKyunKwan University Report New Data on Medical Informatics (Developing a Standardization Algorithm for Categorical Laboratory Tests for Clinical Big Data Research: Retrospective Study). Information Technology Newsweekly.

[10] Sakr, S., Zomaya, A. (2018). Editorial for Special Issue of Journal of Big Data Research on "Big Medical/Healthcare Data Analytics".

[11] (2020). Sanford Burnham Prebys Medical Discovery Institute; New Coronascape tool will help unlock bigdata insights for COVID-19. Medical Letter on the CDC \& FDA. https://www.eurekalert.org/pub_releases/202009/sbpm-nct091520.php. 